\title{
Situación, proceso y construcción de la sociedad de la información en México' ${ }^{1}$
}

\author{
JoEl ESTUdillo García \\ Cen tro de Inves tiga cio nes so bre Amé rica del Nor te (CISAN), \\ UNAM, Torre II de Humanidades, piso 10, \\ CiudadUniversita ria, 04510,México,D.F. \\ E-Mail:ejoel@servidor.unam.mx
}

\section{RESUMEN}

Laépoca actualseca rac te riza por elincremen to delas tec nologías de in formación, las cuales se han convertido en el mo tordelde sa rrolloeco nó mico y so cial. Las ac ti vida des de in for ma ción jue gan un pa pel en el cam bio eco nó mi co dela so cie dad con tem porá nea que tran si ta de una eta pain dus trial a una postindustrial o de información. Este artículo intenta mostrar si en Méxi co está to man do lu gar tal tran si ción del sec tor servi cios al de la so ciedad delain forma ción, paralocual se recopila ron, selec cio na rony analizaron las actividades relacionadas con la información a partir de dos variables: personal empleado en el ámbito de la información y el producto interno bruto generado por éste.

Pa la bras cla ve: So cie dad de la in for ma ción, Produc to in ter no bruto, So cie dad del cono cimiento, Servicios de in for mación.

\section{CONDITION, PROCESS AND CONFIGURATION OF AN INFORMATION SOCIETYIN MEXICO JOEL ESTUDILLO-GARCÍA}

\footnotetext{
ABSTRACT

The present time is charac terized by an in crease in in for ma tion tech nologies that have become the motor of social and economic development. Information activities have had a role in the economic change of the present society which is moving from an industrial stage to a postindustrial or information society. This paper will try to show whether Mex ico is li able to follow this tran si tion from the sec tor services to an information society. Having this in mind, activities related with information were selected and compiled and then analyzed considering two view points: per son nel hired in the in for ma tion field and the gross in ternal product.

Keywords: In for ma tions So ciety; G ross In ter nal Pro duct, So ciety of the Knowledge, Information Services.
}

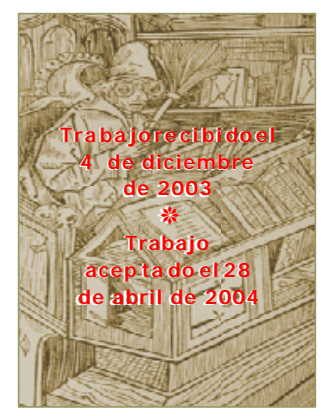

1 Este texto es continuación del artículo "Elementos que conforman la sociedad de la informa ción”, Investigación Biblictedóǵgavol. 15, no.31 (julio-diciembre de 2001): 163-194. 


\section{LA CLASIFICACIÓN MEXICANADEACTIVIDADESYPRO DUCTOS}

Dara analizar el sector económico del país, el Instituto Nacional de Estadística, 1 GeografíaeInformática(INEGI) utilizalaclasificaciónmexicanadeactividadesy productos(CMAP), ${ }^{2}$ que contiene un sis te ma de co di fica ción para agru parda toseconómicos(segúnlas ca tego rías de ac tividad)concaracterís ticas análogas, yquesirve de base para la aplicación de los censoseconómicos en México. La CMAP con tie ne cuatro niveles de agre ga ción: sec tor, sub sec tor, ramay cla se de ac tividad. Esta agrega ción obedece al criterio básico deidentificación dela actividad delas unida desesta dísticas, partiendo de lo general, de manera gradual, a niveles de mayor especificación.

\begin{tabular}{|l|c|c|c|}
\hline \multicolumn{4}{|c|}{ Estructura conceptual de la CMAP } \\
\hline Actividades & Sector & Subsector & Rama \\
\hline Primarias & 1 & & \\
\hline Industriales & 2 a 5 & & \\
\hline Terciarias & 6 a 9 & & \\
\hline Distributivas & 6 y 7 & & \\
\hline Comercio & 6 & & \\
\hline Transporte & 7 & & \\
\hline Inversión & 8 & & \\
\hline Financiamiento & 8 & 1 & \\
\hline Financieras & 8 & 1 & 10 \\
\hline Bursátiles & 8 & 1 & 20 \\
\hline Seguros y finanzas & 8 & 1 & 30 \\
\hline Alquiler de bienes & 8 & 2 y 3 & \\
\hline Otros servicios & 9 & & \\
\hline Administración pública & 9 & 1 & \\
\hline Servicios privados & 9 & 2 a 7 & \\
\hline
\end{tabular}

Fuente: INEGI, Clasificaciónmexicana 1999..., 6.

Para este trabajo se tomaron en cuenta sólo las actividades que se reflejan en el producto interno bruto (PIB) y el número de empleados ocupados y remunerados. Las ac tivida des que se selec cio na ron delaCMAPsees pecifican más adelante; todos losdatosestadísticosseextrajerondeloscensoseconómicos.

"El primercen so eco nó mi co que se rea lizó en México fue en el año de 1930, co ordinadoporlaDirección GeneraldeEs ta dís ticas (DGE). En dis tin tas épo cas, esta dirección funcionó bajo las órdenes de la Secretaría de Economía y la Secretaría de 2 INEGI, Clasificaión mexicana deadividadesyprodudos 1999(Aguas ca lien tes: INEGI, 1999), 7. 


\section{InvestigaaónBiblicteedógica v. 18 N o. 37 julio/ diciembre de 2004}

IndustriayComercio(SIC), llegandoin clu so a depen derdelaPre sidenciadela República(SPP)". ${ }^{3}$ Des de en ton ces, cada cin co años se han rea liza do los cen sos eco nó mi cos en nues tro país (a partirde1930 y has ta el más re ciente de1999).

\begin{tabular}{|l|l|}
\hline \multicolumn{1}{|c|}{ Años } & \multicolumn{1}{c|}{ Institución responsable } \\
\hline 1932-1982 & Dirección General de Estadística \\
\hline 1983-1999 & INEGI \\
\hline
\end{tabular}

Fuen te: INEGI, Direc ción Ge ne ral deEs ta dís tica y Direc ción Técnica, Cien años de la Dirección General de Estadística (México: Dirección Técnica, 1993).

Alanalizarloscensos eco nó micosporlus tros ob serva mos quela cla sifica ciónva ría y no coin ci den los co di fica do res para la mis ma ac ti vi dad, ya que ésta se de pu ra y porlotan toesimposibleseguiruna secuencia es tadís tica re tros pectiva oprospectiva con el mismo clasificador, por ejemplo, el concepto libreńas se representa de la siguientemanera:

\begin{tabular}{|c|c|c|c|c|c|c|c|}
\hline Años & 1961 & 1966 & 1971 & 1976 & 1981 & 1989 & 1994 \\
\hline Codificador & 6551 & 6264 & 6323 & 6227 & 623074 & 623074 & 623074 \\
\hline
\end{tabular}

Como país miem bro de la ONU,Méxicodebeproporcionarlosresultadoscensales conformealaestructura delaClasificaciónIndustrialInternacionalUniforme(CIIU). De igual forma, como país miembro del Tratado de Libre Comercio de América del Norte, tiene quepresentarda tos compatibles conlasesta dís ticas deEs tados Unidosy Canadá. "La adopción del Sistema de Clasificación Industrial de América del Norte (SCIAN)haráposiblecontarconinformaciónnormalizadaconotrospaíses". ${ }^{4}$

\section{EL PROCESO DE CONSTRUC CIÓN DE LASO CIE DAD DE LA INFORMACIÓN EN MÉXICO}

A partir del análisis de los datos proporcionados por los censos económicos del INEGI, Estadísticas Históricas de México y del Banco Nacional de México (Banamex), ela bo ramos un cua dro so bre el nú me rode trabaja do res em plea dos yelPIB gene ra do por cada sec tor eco nó mi co, por lo que se pue de afir mar que en la eco no mía mexicana pre do mi na el sec tor servi cios des de los años se sen ta, ya que más de la mitad de la población económicamente activa (PEA) se dedica a ellos. Los elementos que con forman el sec tor ser vi cios en Mé xi co son edu ca ción, salud, trans portes, ser vicios financieros y telecomunicaciones. La participación del sector industrial

3 INEGI, Méxichy(Aguascalientes: INEGI, 1991), 6.

4 INEGI, Sistema dedasificadónindustrial deAménicaddNorte(México: INEGI, 1999), 16. 
correspondienteal se gun do sec tor eco nó mi conun ca ha sido ma yora 30 porcien to y alcanzó su máximo nivela prin cipios delos no ven ta; tal parti cipa ción nun ca ha sido menora11 porcientodes delaprimeradécadadelsiglo XX.

Lacon tribución delsec torin dus trial alPIB no dejó de cre cerhas ta los años no venta, cuan do se es ta bi li zó en 22 por cien to, por lo que Méxi co nun ca ha sido un país indus trial enel sen ti do de con tarcon una amplia par ticipa ción del PIB y de la fuer za laboralremuneradaenelsectormanufacturero.

Actualmen teen países de sa rrolla doscomo Es tados Unidos, FranciayJa pónlos avances tecnológicossehan apli ca doa la genera cióny dis tribu ción dein forma cióny co munica ción, a tal gra do quelas ac tivida des dein for ma ción porsímis mas han desa rrolla do un cuarto sec toreco nómico, esta me dición sehahe cho conbase en el nú mero de tra baja do res em plea dos yen la ge ne ración delPIBgenera do por tales ac tivida des. Al res pec to, Castells(1995) dicequeactualmenteyasede sarrollansociedadesde lainformaciónyquelospaísesdesarrolladosllevanladelantera.

Porotra parte, en un in forme de la Con feren cia de las Na ciones Uni das so bre ComercioyDesarrollo(UNCTAD, porsussiglas eninglés) semencionaque:

El nuevo motor del crecimiento económico de los países desarrollados ha dejado de ser la energía barata, tomando su lugarlain for ma ción. Sin embargo, el conocimiento y nuevos conceptos de organización son los complementos [de] la información que han permitido que esta revolución científica y tecnológica se concretice en nuevos productos. ${ }^{5}$

LosserviciosenMéxicohansufridounaseriedetransformacionestecnológicasque hancambiadoalgunasdesuscaracterís ticas tradicionales (comosuintensidadenmano de obra y el em pleode nuevas tec no lo gías, por men cio nar sólodos); tam bién se han incrementadolosserviciosproporcionadosporlastelecomunicacionesylainformática.

Estos servicios han permitido elde sa rro llo de nue vas tec nologías enlos países desarrollados, y permiten reducciones con ti nuas en los cos tos la borales de los procesos productivos. ${ }^{6}$

Deon Y.Jeon ana liza este temay co men ta que to das son so cie da des delain forma ción y pue den cla si ficarse como so cie dad delain forma ción alta, me dia y baja. 7 Si retomamos tal idea, México es obviamente una sociedad y por tan to, exis te en ella un sec tor de lain for ma ción, aun que aún no se re pre sen te en los cen sos delINEGI ni en ningu na otra fuen te dees ta dís ticas. Este sec torlocon formanlas uni da des econó mi casquerealizanalguna delas actividadesrelaciona das conlain formación.Laca racterís tica de este sec tor es que las uni da des econó mi cas quelocon forman de sempeñan principalmente activida des relacionadas conlaproduc ción, la ordenación, lacomunica ción olos servicios de in for ma ción. Este sec tor es muy am plio, diverso eim portanteparaeldesarrolloeconómico,socialyeducativodeunasociedad.

\section{UNCTAD, Méxic, una econmía deservicios ReportedlproyedoMEX/ 87/ 026/, 6.}

6 Ilid, 7.

7 Dong Y. Jeon. “The Na tu re of In for ma tion", en Speeial Libranies(verano de 1990). 


\section{InvestigacónBiblictedoógica v. 18 N o. 37 julio/ diciembre de 2004}

En1962,Fritz Ma chlup8intentómedirelporcentajedefuerzalaboralinvolucrada enlabores delconocimien to enEstados Unidos, cuyaac tividad primaria es el trabajo de in for ma ción. Años más tar de, en 1977, Marc Po rat ${ }^{9}$ publicó un trabajo que retomabalainves tiga ción de Machlup, en elque cambióla terminología de conocimiento por la de trabajadoresde la información. Ambasinvestigaciones documentaron un gran y crecien te sec tor de in formaciónen Es tados Unidos. Ensus res pectivasinves tiga cionesparacon formarunsectorde lain formación es tosau to res hi cie ronunaseleccióndeactividadesextraídasdeloscensoseconómicosestadunidenses:

\begin{tabular}{|c|c|}
\hline \multicolumn{2}{|c|}{$\begin{array}{c}\text { Relación y comparación de actividades seleccionadas para analizar } \\
\text { el desarrollo de la sociedad de la información en } \\
\text { Estados Unidos }\end{array}$} \\
\hline Fritz Machlup & Marc Porat \\
\hline Teléfono y telégrafo & Teléfono y telégrafo \\
\hline Máquinas de escribir & Fotocopiadoras \\
\hline Computadoras & Telecomunicaciones \\
\hline Edu ca ción en el ho gar, tra ba jo eigle sia & Teléfono \\
\hline Educaciónes colarelemen talypro fe sional & Telégrafo \\
\hline Escuelas comerciales y vocacionales & Servicio postal \\
\hline Bibliotecas & Editoriales \\
\hline Libros y folletos & Programas de cómputo \\
\hline Periódicos & Educación elemental y profesional \\
\hline Revistas & Profesores particulares \\
\hline Fotografías & Auditorias \\
\hline Radioytelevisión & Administración \\
\hline Telecomunicaciones & Fotógrafos \\
\hline Servicio Postal & Autores \\
\hline Agen tes de bie nes raíces & Radio y televisión \\
\hline Notarías & Finanzas \\
\hline & Gobierno \\
\hline
\end{tabular}

Fuentes: Fritz Machlup, The Production and Distribution of Knowledge in the UnitedStates, 354- 357, 370-371, y Marc Uri Po rat, The InformationEconomy: DefinitionandMeasurement (Washington, D.C.: U.S.: Depart ment of Commerce, 1977).

8 Fritz Ma chlup, TheProductionandDistributionofKnoweelgeintheUniteelStates(Nue va Jer sey: Prin centon Uni ver sity Press, 1971).

9 Marc Uri Porat. TheDifinitionEconamy: DefinitionandMeasurement U.S Department of Comerce, 1977. 
Otroes tudio sodel tema, YonejiMasu da,${ }^{10}$ tam biénclasificólas ac tividades rela cionadas conlain formación en Japón, en trelasquein cluyelas siguientes:

\begin{tabular}{|l|l|}
\hline \multicolumn{2}{|c|}{$\begin{array}{c}\text { Actividades seleccionadas para analizar el desarrollo } \\
\text { de la sociedad de la información en Japón }\end{array}$} \\
\hline Investigadores & Bancos de da tos \\
\hline Escritores & Empresas de sofware \\
\hline Analis tas decrédito & Máquinascopiadoras \\
\hline Investigadoresdeopinión & Filósofos \\
\hline Impresores & Líderes religiosos \\
\hline Fotocopistas & Profetas \\
\hline Encuadernadores & Escuelas \\
\hline Editores & Cursos por correspondencia \\
\hline Reprógrafos & Seminarios \\
\hline Periódicos & Bibliotecas \\
\hline Agencias de noticias & Novelistas \\
\hline Revistas & Compositores \\
\hline Empresas de publicidad & Productoras de cine \\
\hline Relacionespúblicas & Empresas televisivas \\
\hline $\begin{array}{l}\text { Centrosdeprocesamiento } \\
\text { dedatos }\end{array}$ & Equiposfotográficos \\
\hline & Equipos degrabación \\
\hline
\end{tabular}

Fuente: Yoneji Masuda, La sociedadinformatizadacomo sociedad posindustrial.

Si se sigue el ejemplo de estos autores, en México identificamos actividades que conforman el sector de la información. Las actividades, así como su código, los tomamosdela CMAPylaSCIAN(elaboradaporelINEGIen 1998): ${ }^{11}$

10 Yomeji Masuda, La socielad informatizada como soceclad posindustria(Madrid: Fundesco-Tecnos, 1984).

11 Yomeji Masuda, La socedad infomatizada com socedad posindustrial (Madrid: Fundesco-Tecnos, 1984). 


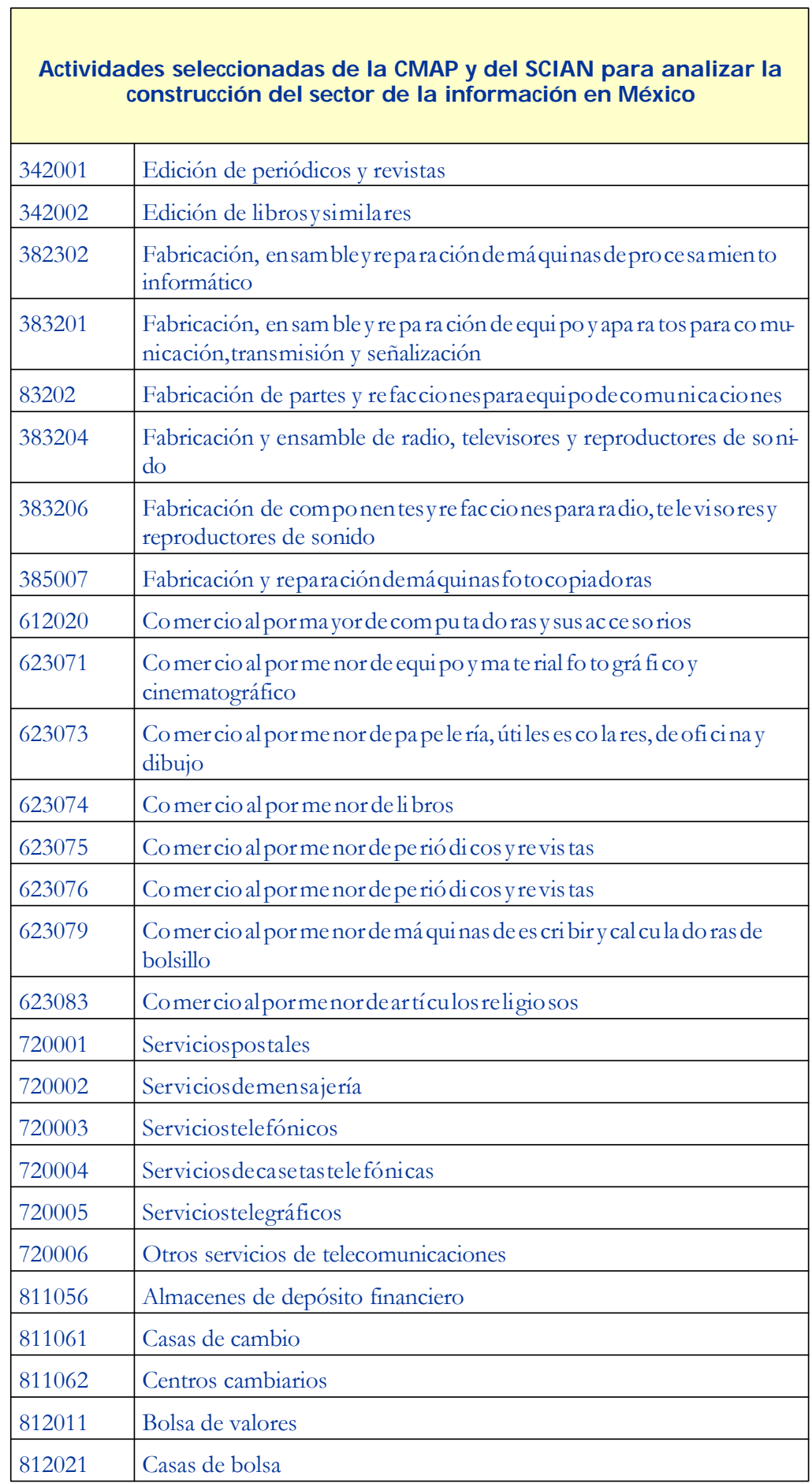




\begin{tabular}{|c|c|}
\hline \multicolumn{2}{|c|}{$\begin{array}{l}\text { Actividades seleccionadas de la CMAP y del SCIAN para analizar la } \\
\text { construcción del sector de la información en México } \\
\text { (Cont.) }\end{array}$} \\
\hline 812031 & Sociedades de inversión \\
\hline 831113 & $\begin{array}{l}\text { Servicio de al quiler de equi po elec tró ni co para el pro ce sa mien to } \\
\text { informático }\end{array}$ \\
\hline 831121 & $\begin{array}{l}\text { Servicio de alquilerdeteleviso res, equipo de so nido, video casetse } \\
\text { instrumentosmusicales }\end{array}$ \\
\hline 831122 & Servicio de alquilerde equipo fo to grá ficopro fe sio naly pro yec to res \\
\hline 921001 & Servicios privados de educaciónpreescolar \\
\hline 921102 & Servicio privado de educación primaria \\
\hline 921103 & Servicios privados de educaciónsecundaria \\
\hline 921104 & Servicios privados de educaciónmediasuperior \\
\hline 921105 & Servicios privados de educaciónsuperior \\
\hline 921106 & $\begin{array}{l}\text { Servicios privados de edu ca ción que com bi nan los ni ve les de en se- } \\
\text { ñanzapreescolar, primaria, secundaria,media superior y superior }\end{array}$ \\
\hline 921107 & Servicios de enseñanza co mercial y de idio mas \\
\hline 921108 & Servicios de capacita ción téc nica, deoficiosyarte sa nías \\
\hline 921110 & Servicios de educación porpartedepro fe so res particulares \\
\hline 921111 & Servicios privados de educaciónespecial \\
\hline 921202 & Servicios públicos deeducación primaria \\
\hline 921203 & Servicios públicos deeducaciónsecunda ria \\
\hline 921204 & Servicios públicos deedu ca ción me dia superior \\
\hline 921205 & Servicios públicos deeducación su pe rior \\
\hline 921206 & $\begin{array}{l}\text { Servicios públicos de edu ca ción que com bi nan di fe ren tes niveles de } \\
\text { enseñanza }\end{array}$ \\
\hline 921207 & Servicios públicos deeduca ción elemental terminal \\
\hline 921208 & Servicios públicos deeduca ción me diaterminal \\
\hline 921209 & Servicios de educaciónpublicaespecial \\
\hline 922100 & Servicios privados de investigación y desarrollo científico \\
\hline 922200 & Servicios públicos deinves tigaciónydesarrollocientífico \\
\hline 941104 & Producción y transmisión privada de programas de ra dio \\
\hline 941105 & $\begin{array}{l}\text { Producción, transmisión y repe tición de programas de tele visión, } \\
\text { servicios privados }\end{array}$ \\
\hline
\end{tabular}




\begin{tabular}{|c|c|}
\hline \multicolumn{2}{|c|}{$\begin{array}{c}\text { Actividades seleccionadas de la CMAP y del SCIAN para analizar la } \\
\text { construcción del sector de la información en México } \\
\text { (Cont.) }\end{array}$} \\
\hline 941106 & $\begin{array}{l}\text { Servicios priva dos de pro mo ción, mon ta je y re pre sen ta ción de es } \\
\text { pectáculos artísticos }\end{array}$ \\
\hline 941107 & Servicio de autores, compositores y otros artistas independientes, \\
\hline 941203 & Emisiones públicas de radioy televisión \\
\hline 942101 & $\begin{array}{l}\text { Servicios priva dos de biblio te cas, mu seos, jar dines bo tá nicosy } \\
\text { similares, }\end{array}$ \\
\hline 942202 & $\begin{array}{l}\text { Servicios públicos de biblio te cas, mu seos, jardines bo tánicosy } \\
\text { similares }\end{array}$ \\
\hline 951001 & Servicios deno tarías públicas \\
\hline 951002 & Servicios debufetesjurídicos \\
\hline 951003 & Servicios de con ta du ríay au di to ría \\
\hline 951004 & Servicios de análisis de sis te mas y proce sa mien to in formático \\
\hline 951005 & Servicios de ase so rías y es tu dios téc ni cos de in ge nie ría y ar qui tec tu ra \\
\hline 951006 & Servicios de diseñoindustrial \\
\hline 951007 & Serviciosdemercadotecnia \\
\hline 951014 & Servicios deagen cias noticiosas \\
\hline 951009 & Servicios de diseñoartís tico \\
\hline 951010 & Servicios de ase so ría en ad mi nis tra ción y or ga ni za ción de em pre sas \\
\hline 951013 & Servicio de investigacióndesolvenciafinanciera \\
\hline 951014 & Servicios de agen cias noticiosas \\
\hline 951016 & Servicio de revelado de fotografía y películas \\
\hline 951017 & Servicios de fo to copia do y simila res. \\
\hline 952002 & Servicio de fotografía \\
\hline 952007 & Servicio de predicción del futuro \\
\hline
\end{tabular}

En Méxicoes tosservicios in cluyenalas bibliote cas en sus diversas modalidades: publicas, privadas, especializadas, universitarias, así como centros de documentacióneinformación.

Existen también servicios de información co mer cial, los cua les ofre cen pro ductoscomodirectoriosycatálogosensus diferentes variantes: telefónicos, indus triales, comerciales, catálogosdeproductosydeservicios, entreotros. 
Con los datos que Porat recopiló de los censoseconómicos de Estados Unidos, representó estadísticamenteelnacimien todeuncuarto sectoreconómicoensupaís, conbase en dos va riables: personal emplea do yproduc toin ternobru to ge ne rado en lasactividadesrelacionadasconlainformación.

\section{Personal ocupado por sectores económicos en Estados Unidos}

\begin{tabular}{|c|c|c|c|c|}
\hline Año & Agricultura & Industria & Servicios & Información \\
\hline 1900 & 35.3 & 26.8 & 25.1 & 12.8 \\
\hline 1910 & 31.1 & 36.3 & 17.7 & 14.9 \\
\hline 1920 & 32.5 & 32 & 17.8 & 17.7 \\
\hline 1930 & 20.4 & 35.3 & 19.8 & 24.5 \\
\hline 1940 & 15.4 & 37.2 & 22.5 & 24.9 \\
\hline 1950 & 11.9 & 38.3 & 19.0 & 30.8 \\
\hline 1960 & 6 & 34.8 & 17.2 & 42.8 \\
\hline 1970 & 3.1 & 28.6 & 21.9 & 46.4 \\
\hline 1980 & 2.1 & 22.5 & 28.8 & 46.6 \\
\hline
\end{tabular}

Fuen te: Marc Po rat, "Por cen taje de la fuer za de tra bajo por sec tor eco nó mi co en Estados Unidos: 1900-1980".

Alre to maresteartículohicimoslomis mo para Méxicoa fin de represen tarelpersonal empleado y la generación del PIB enactividades relacionadascon la información. Los datos estadísticosrecopilados para confor marun sec torvin cula do con la in formaciónenMéxico em pie zan are gis trarseenlos añossesenta, datoque coincide conlo ex pues to por Po rat, Bell y Ma su da. A partir de esta fe cha seplas man ac tivida des rela tivas ala in for ma ción enlos cen sos econó mi cos co rres pon dien tes a co mercio, in dustria, servicios, transportesycomunicaciones. Acontinuaciónpresentamos los censos consultados, el número de codificador de las actividades y las fechas en quesepublicarondichoscensos. 


\begin{tabular}{|c|c|c|c|c|c|}
\hline \multicolumn{6}{|c|}{$\begin{array}{c}\text { Censos económicos utilizados para obtener datos relacionados con el sector } \\
\text { de la información en México }\end{array}$} \\
\hline VII Censo económico 1961 & $\mathrm{x}$ & & CMAE & 4 dígitos & SIC, Direc ción Generalde Es ta dís tica \\
\hline VIII Censo económiø1966 & $\mathrm{X}$ & & CMAE & 4 dígitos & SIC, Direc ción GeneraldeEs ta dís tica \\
\hline IX Censoeconónico 1971 & $\mathrm{X}$ & $\mathrm{X}$ & CMAE & 4 dígitos & SIC, Direc ción GeneraldeEs ta dís tica \\
\hline X Censoeconónico1976 & $\mathrm{X}$ & $\mathrm{X}$ & CMAE & 4 dígitos & $\begin{array}{l}\text { Secretaría de Programación y Pre su- } \\
\text { puesto, Coordinación Ge ne ral de Sis- } \\
\text { tema NacionaldeInformación }\end{array}$ \\
\hline XI Censoeconónico 1981 & \multicolumn{5}{|c|}{ No existe información } \\
\hline XII Censoeconómico1986 & $\mathrm{X}$ & $\mathrm{X}$ & CMAP & 6 dígitos & INEGI \\
\hline XIII Censoeconómico1989 & $\mathrm{X}$ & $\mathrm{X}$ & CMAP & 6 dígitos & INEGI \\
\hline XIV Censoeconómiœ1994 & $\mathrm{X}$ & $\mathrm{X}$ & CMAP & 6 dígitos & INEGI \\
\hline XV Censoeconómico1999 & $\mathrm{X}$ & $\mathrm{X}$ & SCIAN & 6 dígitos & INEGI \\
\hline
\end{tabular}

CMAE: Clasificaciónmexicanadeactividadeseconómicas.

CMAP:Clasificaciónmexicanadeactividadesyproductos.

SCIAN: SistemadeClasificaciónIndustrialdeAméricadelNorte.

\section{El PIB en México}

Mediante el análisis de la concentración de datos estadísticos recabados desde 1930 se ob serva que en México pre dominó una eco no mía de servicios, aun que bá sicamen te se refería al co mercio y no a servicios rela cio na doscon el proce sa mien to de información. La generación del PIBen este sec tor siem pre es tu vo poren ci ma de los sectoresagrícolaeindustrial.

A me dia dos de los años cin cuen ta, se apre cia un au men to delPIB en el sec tor industrial que al ser comparado con el sector agrícola desde esa fecha hasta nuestros días, mues tra que elPIB enelsectoragrícolasehamanifestadoalabaja.

Por otra parte el sector industrial mantuvo su predominio solamente durante la épo ca delos años se ten ta, que fue cuan do al can zó un nivel rela ti va men te alto y reba sóal sec tor agrícola. Du ran te los años ochen ta y no ven ta, su cre cimien to no refle jó grandescambios.

Encuan to al sec tor servicios éste siem pre ha man te ni do una hege mo nía muy alta en com pa ra ción con los sec to res agrí co la ein dus trial: des de fines delos años ochenta, este sector ha cre ci do a un rit mo ace le ra do e in clu so ha ge ne ra do más de 60 por cientodel PIBparalaeconomíamexicana. 
PIB en México por sectores de actividad económica (1910-1994)

\begin{tabular}{|c|c|c|c|}
\hline Años & Sector primario & Sector secundario & Sector terciario \\
\hline 1910 & 12.9 & 12.3 & 50.7 \\
\hline 1921 & 35.2 & 11.0 & 53.0 \\
\hline 1930 & 29.0 & 16.1 & 55.0 \\
\hline 1940 & 25.0 & 17.7 & 55.5 \\
\hline 1950 & 24.1 & 21.3 & 54.6 \\
\hline 1960 & 20.6 & 24.0 & 55.3 \\
\hline 1970 & 16.2 & 29.3 & 54.5 \\
\hline 1980 & 19.1 & 19.2 & 59.3 \\
\hline 1985 & 12.0 & 27.2 & 60.0 \\
\hline 1990 & 11.2 & 29.0 & 60.3 \\
\hline 1994 & 10.6 & 29.1 & 60.4 \\
\hline
\end{tabular}

Fuen te: Censos generalesdepoblación, 1900-1950 y 1960; Se cre ta ría deProgramaciónyPresupuesto;DirecciónGeneraldeEstadística;INEGI;Dirección General de Contabilidad Nacional, Estudios socioeconómicosy precios; Dirección de Contabilidad Nacional, "Sistema de cuentas nacionales de México" (varios años) y se lec ción de da tos es ta dís ti cos so bre la eco no mía me xi cana, en NacionalFinancieray Cenedic,Laeconomíamexicanaencifras (México: NacionalFinanciera, 1995).

Encuan to al sec tor delain forma ción, los da tos co rres pon dien tes alPIB empezaron a consignarse en los censos económicos a par tir de 1970; an tes de esa fe cha no existendatosregistradosenloscensoseconómicosdeMéxico.

\begin{tabular}{|c|c|}
\hline \multicolumn{2}{|c|}{ PIB generado en el sector de la información en México } \\
(millones de pesos) \\
\hline Año & Total \\
\hline 1971 & 8,930 \\
\hline 1976 & 18,016 \\
\hline 1981 & 41,611 \\
\hline 1986 & 998,515 \\
\hline 1989 & 11,293 \\
\hline 1994 & 50,765 \\
\hline 1999 & 532,575 \\
\hline
\end{tabular}




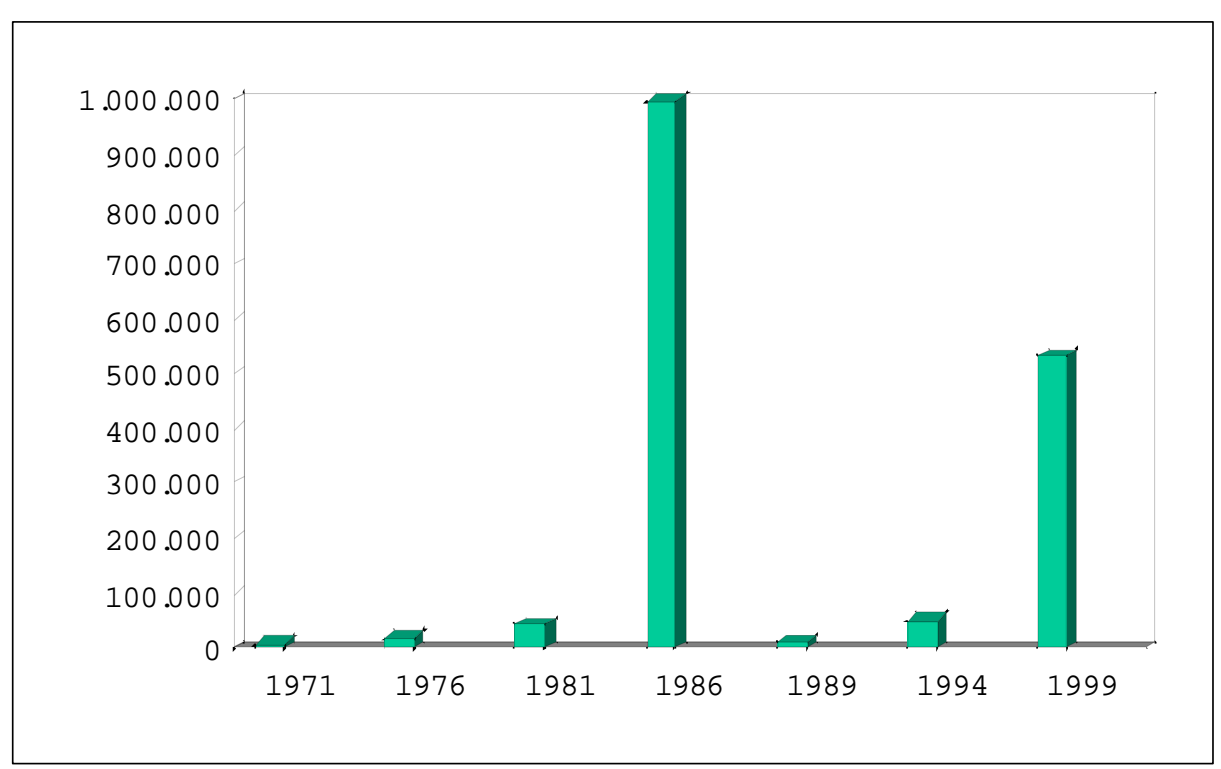

Las fuen tes em plea das para ob te ner in for ma ción fue ron cen so co mer cial, in dus trial, servicios, comunicaciones y transportes. En 1981 no se registrarondatos del PIB en el censo de servicios. Para uni for mar los da tos, todo se con vir tió a mi les de pe sos.

\section{El PERSONALOCU PAdO EN MÉXico}

Un análisis de la con cen tra ción de da tos es ta dís ticos so bre perso nalocu pa do re cabadodes deprincipios delsigloXX, mues trauncomportamien todiferentedelPIB, ya que des de 1900 has ta 1970 el por cen taje más alto de em plea dos se dio en el sec tor económicoagrícola.

El sector industrial tuvo un incrementoconsiderable a partir de la década de los años se sen ta ylle góa sumáximonivelenlos se ten ta yochen ta, perode cre ció enla década siguien te. Este sec tor nun caigua ló ni re ba só al per so nal em plea do en el sec tor agrícola,y tampocoestuvoporencimadelsectorserviciosduranteelsiglopasado.

En cambio el PIB del sector servicios, que estuvo por arriba del sector agrícola desde1900 has ta1960, semantuvomuy pordebajodelascifras depersonalocu pado enelsectoragrícolaenestemismoperiodo.

A par tir de los años se ten ta se apre cia que el nú me ro de em plea dos au men ta en el sector servicios de jan do por de ba jo el por cen ta je del sec tor agríco la; a par tir de entonceselnúmerodeempleadossiemprehasidomás elevadoenelsectorservicios. 


\section{Ocupación de la PEA en México por sectores de actividad económi- ca (1900-1993)}

\begin{tabular}{|c|c|c|c|}
\hline Año & Primario & Secundario & Terciario \\
\hline 1900 & 77.2 & 15.8 & 7 \\
\hline 1910 & 75.7 & 16.9 & 7.5 \\
\hline 1920 & 78.2 & 14.1 & 7.7 \\
\hline 1930 & 76.9 & 15.1 & 8 \\
\hline 1940 & 65.4 & 15.6 & 19 \\
\hline 1950 & 58.3 & 15.9 & 25.8 \\
\hline 1960 & 54.2 & 19 & 26.8 \\
\hline 1970 & 34.7 & 21.2 & 44.1 \\
\hline 1975 & 30.4 & 22.1 & 47.5 \\
\hline 1980 & 29 & 22 & 49.1 \\
\hline 1985 & 28.9 & 20.5 & 50.5 \\
\hline 1990 & 26.7 & 22.3 & 51 \\
\hline 1993 & 26.6 & 22.1 & 51.3 \\
\hline
\end{tabular}

Fuen te: Censos generalesdepoblación, 1900-1950 y 1960; Se cre ta ría de Programacióny Pre su puesto; Direc ción GeneraldeEs ta dís tica; INEGI; Dirección General de Contabilidad Nacional, Estudios socioeconómicos y precios; Dirección deContabilidadNacional, "Sistemadecuentasnacionales deMéxico" (varios años) y se lec ción de da tos es ta dís ti cos sobre la econo mía mexicana, en NacionalFinancierayCenedic,Laeconomíamexicanaencifras (México: NacionalFinanciera, 1995).

Encuan to al per so nal em plea do en el sec tor de la in for ma ción a partirde los años se sen ta y has ta me dia dos delos noven ta, según los da tos to ma dos delos cen sos econó mi cos de Mé xi co, se apre cia que no hay una con ti nui dad de bi do a que al gu nos de los censoseconómicos consul tados no contabanconda tos debido acircunstan cias diversas; sinembargo, se advier teque este sec torha emplea doaun mayor nú me rode personasdesde1960alafecha.

\begin{tabular}{|c|c|}
\hline \multicolumn{2}{|c|}{$\begin{array}{c}\text { Personal ocupado en el sector de la información en } \\
\text { México (1960-1994) }\end{array}$} \\
\hline Año & Total \\
\hline 1961 & 54,767 \\
\hline 1966 & 125,111 \\
\hline 1971 & 499,138 \\
\hline 1976 & 131,238 \\
\hline 1981 & 416,719 \\
\hline
\end{tabular}


70 Investigacón Biblidtedoógica v. 18 N o. 37 julio/ diciembre de 2004

\begin{tabular}{|c|c|}
\hline \multicolumn{2}{|c|}{$\begin{array}{c}\text { Personal ocupado en el sector de la información en } \\
\text { México (1960- 1994) } \\
\text { (Cont.) }\end{array}$} \\
\hline Año & Total \\
\hline 1986 & 451,366 \\
\hline 1989 & 607,216 \\
\hline 1994 & 863,974 \\
\hline 1999 & $1,649,167$ \\
\hline
\end{tabular}

PersonalocupadoenelsectordelainformaciónenMéxico

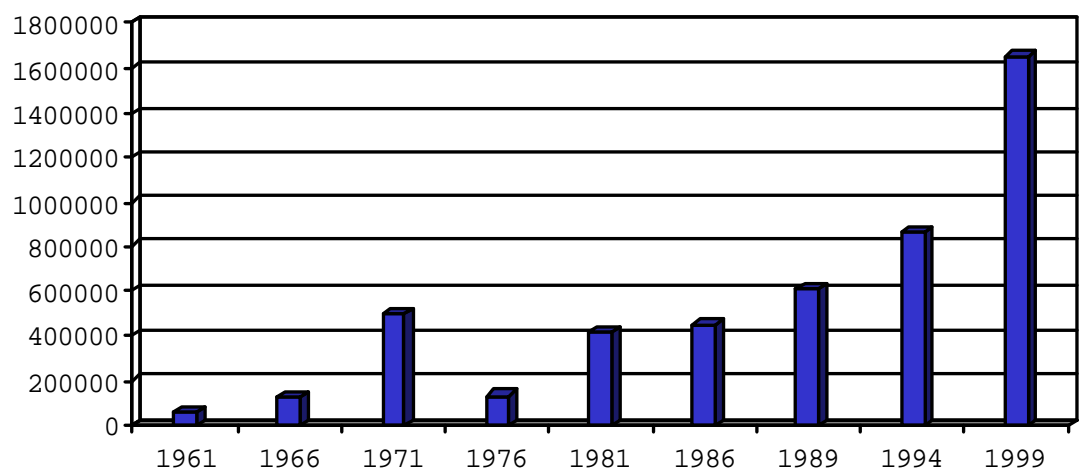

Los da tos se ob tu vie ron de los cen sos de ser vi cios, in dus trial, co mercial y trans por te. El censo de comunicaciones y transportes comenzó a registrar da tos del per so nal ocu pa do a partir de 1981.

\begin{tabular}{|c|c|c|c|c|}
\hline \multicolumn{5}{|c|}{ Personal ocupado por sector económico } \\
en México
\end{tabular}

Fuen te: INEGI,Dirección GeneraldeContabilidadNacional,Estudios Socioeconómicosyprecios; DireccióndeContabilidadNacional,Sistemadecuentasnacionales. 


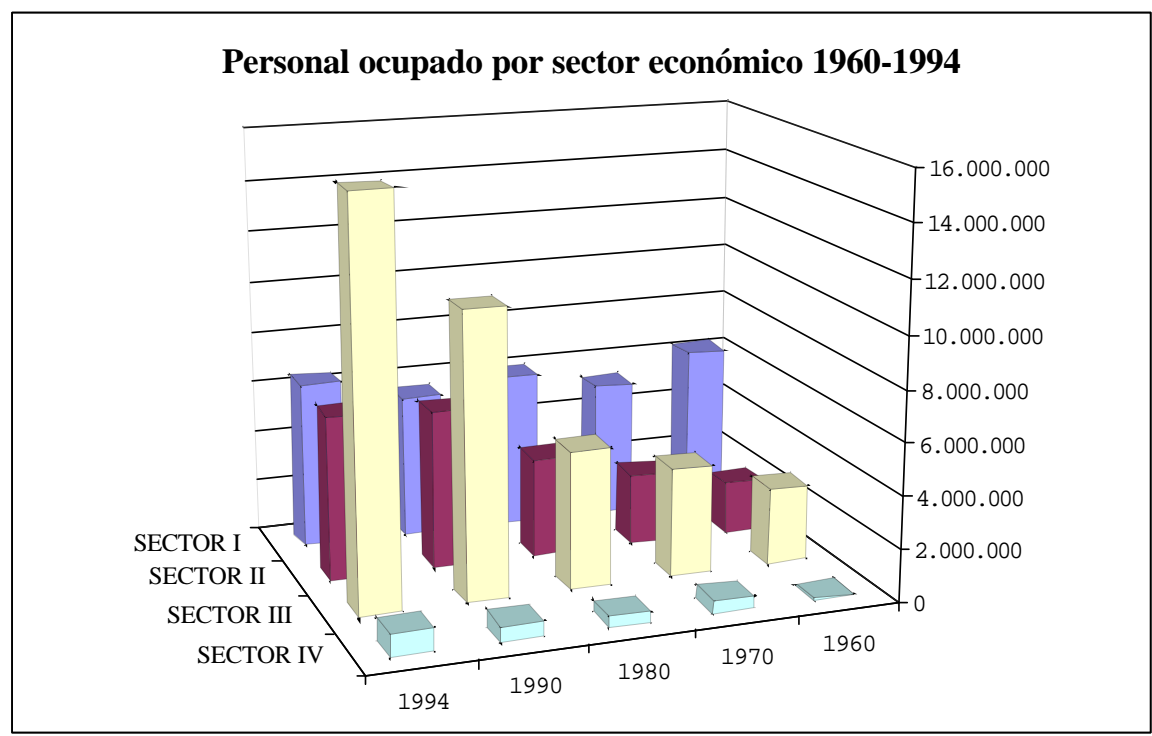

Conlos datosobtenidos delos censos económicos sobrepersonalocupadosepueden represen targrá ficamen teloscua tro sec to reseconó micos yob servarcómosema nifiesta su crecimien to des de los años sesen ta has ta me dia dos delosnoventa, cosa que no se pudo rea li zar con elPIB, porque hay mu chava riación enlascan tidades debidoala ines ta bili dad denues traecono míay ala devalua ción del peso. También sepue dehacer unexamen deldesa rrollodelsector dein forma ción y co munica ción apartirdeldis cursopolíticoenel que se ha manifesta doque el PoderEje cutivo haimpulsa doy desarrolla do las ac tivida des de in for ma ción y co muni ca ción en nues tro país porcon side ratto un sector de gran importancia para la generación de empleos, el mejoramiento de la educaciónycomoproductordeunaltoporcentajedelPIB.

Enlosin formes se observa laim por tan cia que tie ne el Plan Na cional deDe sa rrollo Informático al cual dan seguimiento los me dios dein formación ycomu nicación,yes visiblelapreocupación delEstadoporin crementarmás elpresupues to para eldesarrollo del plan. Un pun to en el que se pone mu choén fa sis en es tos in for mes, a prin cipios delos noven ta, esla pues ta enórbi ta delos sa téli tes MorelosIyII,y pos te riormente el proyectodelossatélitesSolidaridad.Semanifiestalaimportanciaeconómicaque exis te sobrelos servicios dein formacióny comunicación,enlosqueelgobierno acaparapara suspropiosfinesunagran potencialidaddelas funcionesquerealizan es tos sa télites debidoasucarácterestratégicoydedicaelrestoalaventadeconcesiones privadas.

A partirde 1989 advertimos que sele co mien za a dargran im portan cia al sec torde lastelecomunicaciones conlaelaboracióndelPlan NacionaldeDesarrolloIn formá tico, el cual hace hincapié en que las telecomunicaciones son de gran valor para la 


\section{Investigacón Biblictecoógia v. 18 N o. 37 julio/ diciembre de 2004}

eco no mía de México y para el de sa rro llo de otras ac tivida des rela cio na das con lain formación,lasalud,laeducaciónetcétera.

Como dato adicional de este apartado cabe señalar que en la actualidad, Estela Morales, investigadora del Centro Universitario de Investigaciones Bibliotecológicas, es una de las especialistas que se ha abocado a este tema desde una perspectiva académica, y existe el Plan de Desarrollo Informático 1995-2000 como guía, para darleseguimientoanuestrodesarrolloinformacional.

\section{LACONST RUC CIÓN DE LASO CIE DAD DE LAIN FORMACIÓN EN MÉXICO}

Para de sa rrollareste temanos ba sa mos en da tos his tó ricosque ofre cenpau tas de análisisyconstruccióndenuestrasociedadconlos siguientes elementos:

* Selección de las actividades de información y comunicaciones.

* Clasificacióndelasactividadesdein formación(in fraestructurabasadaentecnologías de la información y comunicación).

* Cambios en la estructura económica a partir del análisis estadístico del INEGI.

SegúnJeon, "[...] exis ten dos al terna tivas para po deranalizarla es truc tu ra delsec torin for ma ción: la pri me ra es el aná li sis de la es truc tu ra de la fuerza de tra bajo en la información (análisis ocu pacio nal) y la de riva da de ar tículos y servicios dein formación (análisis industrial)."12 De don de de du ci mos quelas so cie da des de la in for ma ción se insertan en un proceso internacional de transformación mediante el uso de tecnologías dein formacióny porelnúmerodepersonas ocu pa dasquetrabajanental sector. "Los países desarrolla dos oen vías de de sa rrollo para trans formarseen so ciedades dein forma ción de be ránutilizarlain for ma ción como un me dio de mejorar su competitividad o, en su defecto, de conservar su posición en un entorno mundial cadavezmáscompetitivo". ${ }^{13}$

México, al igual que otros países, ha tomado con cien cia de la im portan cia y utilidad de la edu ca ción, la cien cia y la tec no lo gía como he rra mien tas efica ces en el de sa rrollo socioe conómi coy, en con secuencia, in tenta disminuirsu de pendencia depá ses de sa rro lla dos y redu cirla bre cha exis ten te en comparación con éstos. "Enpaíses la tino ame rica nos como México, elEs ta do ya ha empeza do a dar res pues tas a su respon sa bi li dad de pro veer de in for ma ción al pueblo, como parte delos proce sos edur cativosyculturales". ${ }^{14}$

En lo que se refiere al desarrollo de nuevas tecnologías de información, el Poder EjecutivomexicanoelaboróelPlandeDesarrolloIn formático1995-2000,enelquese muestra el interés y preocupación porque nuestro país desarrolle una sociedad de la

12 Jeon, "The NatureofInformation...".

13 Unesco, Repartemmdialsobrelainformadón, 1997-1998, 289.

14 Es tela Mo ra les Cam pos, co ord.,Lainformadónendinidodklaeadectrónica(Mé xi co:CUIB, UNAM, 1998), 24. 
información. "Es ta mos vivien do un cam bio ha cialo que ya se co no ce comola so ciedaddelainformación dela cuallainformática eslainfraes tructura fundamental". ${ }^{15}$

El progra ma pro poneque para ob te nerel máximoaprovecha mien to dela in formá tica en Méxi co se requiere,poruna parte, es timularelusodelas tec nologías deinfor ma ción en los dis tin tos sec to res del país y, por la otra, con tarconla in fraes truc tura requerida: profesionales de la información, investigación y desarrollo tecnológico, industrial,yredes decomunicación, entreotros.

Paraqueelprogramaseamensurable, seestablecenseisobjetivosgenerales:

1. Promover elaprove chamien to dela in formática en los sec to res público,priva do y social del país.

2. Impulsar la forma ción de re cur sos hu ma nos y el de sa rrollo dela cul tu rain formática.

3. Estimular la investigación científica y tecnológica en informática.

4. Fomentar el desarrollo de la industria informática.

5. Propiciar el desarrollo de la infraestructura de redes de datos.

6.Consolidarinstancias decoordinacióny dis posiciones ju rídicas adecuadas para la actividad informática. ${ }^{16}$

LoestipuladoenelPlandeDesarrolloInformáticopermiteverelinterésquetiene nues tro país en de sa rrollaruna socie dad delain for ma ción, que ya exis te en países desarrollados.

La tecnología de informaciónla tinoamericanahades tacadoeneldiseño de soft wares de uso lo cal o re gio nal, y en la ela bo ra ción deCD-ROM por BIREME (Biblioteca Regional de Medicina, con sede en Brasil) y por el Cenedic de la Universidad de Colima, que elabora varios discos latinoamericanos. ${ }^{17}$

Debido a que la evolución, globalmente, se perfila hacia sociedades informacionales, México no debe de jara la de riva el de sa rro llo del sec torin forma cional, y aun cuandono es tamos pre para dos para competirenuna es cala mundialsícon ta moscon las con diciones paradesa rrollarredes dein formaciónmediantelasuniversidades públicasyprivadas, eindustrias como'TelmexyCondumex, entreotras.

Hoylas nuevas tec nologías dein forma cióny co mu nica ciónseemplean paralas más diversas actividades (educación, investigación, aprovechamiento de recursos, prestacióndeservicios bá si cos, co mercio, finanzas, etcé te ra). Elsec torpú blicopuedemejorar significativamente su funcionamiento y productividad, además de atender las demandas prio rita rias en el sis tema edu ca tivo, los servicios de salud, la se gu ridad so cialy laseguridadpública,entreotras.

15 Poder Ejecutivo Federal, Plan Nacianal deDesamdlo 1995-2000. Progama deDesamdlo Informático (México,Secretaría de Hacienda y Crédito Publico), 4.

16 Ibid, 13.

17 Unesco, Repatemmdial sobrelainformadón, 1997-1998, 112. 


\section{Investigacón Biblictecoógia v. 18 N o. 37 julio/ diciembre de 2004}

En el sectorpriva doel aprovechamien todelain formáticaes fundamental parasu desarrollo; porejemplo, elsec torfinancieroeselprincipalconsumidorderecursos en Méxicodebidoaquerequiereprocesargran desvolúmenes deinformacióny tambiéna lagrandependen ciaque tienenlos serviciosbancarios delatecnologíain formáticay las telecomunicaciones.

Las indus trias au to motriz, deau topartes y ma quila dora tam bién hacenusodelas nuevas tecnologías de la información para concluir procesos potencialmente peligrosos. "La educación, investigación, aprovechamiento de nuestros recursos, la prestación deserviciosbásicos, el funcionamien to delcomercio, las finanzas, lossecto resproductivos yelgo biernoseapoyannecesariamenteenlastecnologías dein formación, que yasonelsignodenuestrostiempos". ${ }^{18}$

Especialistascomo FedericoKuhlmann consideran que el desarrollo de una socie dad delain forma ción debeplan tear se cier tos ele men tos rela ciona dos conlasitua ción de mo grá fica, eco nó mi ca y so cial de un país. La de man da de servicios de co mu nicación einformacióntienequeaso ciarse confenómenos demográficostalescomo laes truc tu radelapobla ción, losgru pos de eda desylaurbaniza ción; y asun tos educativos y económicos como la distribución y el nivel de ingresos o la importancia de ciertossectoresquerequierenserviciosdeinformación.

En el rubro educativo el nú me ro de anal fa be tas ma yo res de diez años des cen dió considerablementeen nues tro país en las úl ti mas cua tro dé ca das en tér minos rela tivos, y segúnestimaciones de Fe de ri co Kuhl mann, más del 90 por cien to de nues tra poblaciónesalfabetayhacrecidola matrícula dees tu diantesenlosnivelesmedio superiorysuperior.

\begin{tabular}{|c|c|c|c|c|}
\hline \multicolumn{5}{|c|}{ Población escolar en educación superior (1980-1998) } \\
\hline Años & $\begin{array}{c}\text { Licenciatura } \\
\text { Universitaria y } \\
\text { tecnológica }\end{array}$ & $\begin{array}{c}\text { Educación } \\
\text { normal }\end{array}$ & Posgrado & Total \\
\hline 1980 & $731147 *$ & 96590 & 25503 & 853384 \\
\hline 1981 & 785419 & 112307 & 32139 & 929865 \\
\hline 1982 & 840368 & 125860 & 32169 & 998397 \\
\hline 1983 & 879240 & 133877 & 32970 & 1046087 \\
\hline 1984 & 939513 & 132163 & 35390 & 1107066 \\
\hline 1985 & 961468 & 125236 & 37040 & 1123744 \\
\hline 1986 & 988078 & 131500 & 37955 & 1157533 \\
\hline 1987 & 989414 & 132304 & 38214 & 1159932 \\
\hline
\end{tabular}

18 ErnestoZedillo PoncedeLeón, "Dis cursodelpresidentedela RepúblicaErnes toZedillo, du rantela ceremonia deins tala ción dela Co misión NacionaldeConversiónInformática año 2000, 3 de ju lio de 1998, en Los Pi nos” (Méxi co: Pre si den cia de la Re pú blica, 1998), 7. 


\begin{tabular}{|c|c|c|c|c|}
\hline \multicolumn{5}{|c|}{ Población escolar en educación superior (1980-1998) } \\
(Cont.)
\end{tabular}

Fuente: Anuarios es tadís ti cos de la ANUIES, 1980-1998.

La dis tri bu ción geo grá fi ca de la po bla ción tam bién in flu ye en la de man da de comu nica ciones; en nues tro país se han de sa rrolla do parale la men te otros dos cen tros urbanos degranimportancia:Monterreyy Gua dalaja ra, la fronteranorteigualmente hatenidouncrecimien to poblacional, particularmenteenlafronteraconCalifornia. Ladis tribucióngeográfica dela población nacional seguramenteseguirámodificándoseenelfuturocercano.

\begin{tabular}{|l|c|c|c|}
\hline \multicolumn{4}{|c|}{$\begin{array}{c}\text { Población urbana nacional y de las diez principales } \\
\text { áreas metropolitanas }\end{array}$} \\
\hline \multicolumn{1}{|c|}{ Zona Metropolitana } & $\mathbf{1 9 5 0}$ & $\mathbf{1 9 7 0}$ & $\mathbf{1 9 9 0}$ \\
\hline Poblaciónurbana total & $10,983.000$ & $28,309.000$ & $57,960.000$ \\
\hline Grado de urbanización & 42.6 & 58.7 & 71.3 \\
\hline Diez principales zonas metropolitanas & $4,844.000$ & $14,684.000$ & $26,801.000$ \\
\hline CiudaddeMéxico & $3,137.000$ & $8,904.000$ & $15,048.000$ \\
\hline Guadalajara & 380.000 & $1,493.000$ & $2,987.000$ \\
\hline Monterrey & 339.000 & $1,248.000$ & $2,604.000$ \\
\hline Puebla & 234.000 & 732.000 & $1,330.000$ \\
\hline León & 157.000 & 470.000 & 982.000 \\
\hline Toluca & 115.000 & 373.000 & 904.000 \\
\hline
\end{tabular}




\begin{tabular}{|l|c|c|c|}
\hline \multicolumn{4}{|c|}{$\begin{array}{c}\text { Población urbana nacional y de las diez principales } \\
\text { áreas metropolitanas } \\
\text { (Cont.) }\end{array}$} \\
\hline \multicolumn{1}{|c|}{ Zona Metropolitana } & $\mathbf{1 9 5 0}$ & $\mathbf{1 9 7 0}$ & $\mathbf{1 9 9 0}$ \\
\hline Ciudad Juárez & 123.000 & 407.000 & 789.000 \\
\hline Torreón & 147.000 & 483.000 & 792.000 \\
\hline Tijuana & 60.000 & 277.000 & 699.000 \\
\hline San Luis Potosí & 152.000 & 297.000 & 666.000 \\
\hline
\end{tabular}

Porcentaje depoblación que viveen localidades de más de 2,500habi tan tes res pec toa la po blación nacional. Fuente: INEGI, X Censosgenerales de población y vivienda, 1950, 1970 y 1990.

La mayor par te delos servi cios de co mu nica cio nes se aso cia con fe nó me nos demográ ficos, por ejem plo, la es truc tura de po bla ción por gru pos de eda des, la ur bani zaciónoasun tos econó micos comoelniveldeingresosydemás.

Elprocesodeurbanización deMéxicosecaracteriza porunacrecienteconcentra ción dela pobla ción en unos cuan tos cen tros urba nos, prin ci palmen te enlazona metropolitanadelaciudaddeMéxico.

Esto explica en buena medida que los servicios de comunicaciones nacionales hayan evolucionado siguiendo una configuración tipo estrella, con su cen tro en la ciu dad de Mé xi co, he cho que sin duda se ha vis to fa vo re cida por serésta el asen ta mien to delos po de res fe de ra les y el ma yor centro cultural y financiero, industrial y comercial del país. ${ }^{19}$

En otras palabraslage nera ción delPIBnacionalseguiráal tamentecon centradaenla zona cen tro del país y, por ende será en di cha zona don de se em plea rá a un nú me ro ma yor de perso nas, eigual men te se ge ne ra rála de man da más im por tan te de servi cios de comunicación, apartedequeseemplearáahíaunmayornúmerodeprofesionis tas.

Como se ob servó an tes, si se ma ni fies ta un in cre men to en elPIB relacionadocon el sector de la in forma ción, exis ti rá un ma yor nú me ro de em plea dos ocu pa dos y re mu ne rados enlas áreas vin cula das conlain for ma ción, y tam bién ha bráun ma yornút merodeestudiantesyprofesionistasqueutilicenserviciosdeinformación.

Con los da tos has ta aquíanaliza dos, se ad vier te que en Méxi co se está cons tru yendo un importan te sec tor delain forma ción, aun que tal pro ce so sea de sigualen trelos sec to res dela po bla ción. La so cie dad delain for ma ción seca rac te riza por serelfenóme node trans for ma ción so cial surgi do en tor noal de sa rrollo delas tec no lo gías dela información y comunicaciónen las úl timas cua tro dé ca das. Elimpac to de es tas tecnologías hasido tras cen den te porque prác tica men teno exis te dis cipli naal gu na enla que su pre sen cia no sea pal pa ble y no haya provo ca do una fuer te trans for ma ción en lasactividadessociales, culturalesyeconómicas.

19 Federico Kuhlmann, Comuicadiones pasadbyfuturos 66. 
Colín Clark (1940) dividió el de sa rro llo delas so cie da des a partir de tres pers pectivas: 1 . esen cialmen te agríco la,2.in dus trialy 3.pos tin dus trial, esta úl tima co rrespondea la eta pa his tó rica en la quelos hom bres seliberandel trabajo ma nual yco mienzan autilizar el trabajoba sadoen el conocimien toylain forma ción. Posteriormente, retomaría la idea Daniel Bell, Manuel Castells, Marc Porat y otros, quienes aseguran que todas las sociedades recorrerán estas fases hasta llegar a conformar un cuarto sector: elpostindustrialodelinformacionalismo.

La con for ma ción de un sec tor de la in for ma ción, jun to a los sec to res de pro ducción (primario, secundario y de servicios) les permite a estos estudiosos establecer periodos históricos y determinar el paso de las sociedadesindustriales hasta constituirse en sociedades postindustriales, las que se distinguen porque en ellas la creación, ma nipula cióny co munica ción de in forma ción tie ne prima cía so brela pro duccióndebienesmaterialesyservicios.

Ac tual men te en Mé xi co nos en con tra mos en la terce ra edad o en el sec tor servicios, en el que las actividades eco nó mi cas se dividen en dos ru bros: 1) pro duc to de bienes y servi cios y 2) la in forma ción, esteúl ti mo aún nolo es tu dia por se pa ra do el INEGIensuses tadísticas; sinembargo, estas actividades sehanincrementadoenlos diversoscensoseconómicos.

\begin{tabular}{|c|c|c|c|c|c|c|c|c|}
\hline \multicolumn{7}{|c|}{ Actividades seleccionadas para la construcción del sector de la } \\
información en México \\
\hline $\mathbf{1 9 6 1}$ & $\mathbf{1 9 6 6}$ & $\mathbf{1 9 7 1}$ & $\mathbf{1 9 7 6}$ & $\mathbf{1 9 8 1}$ & $\mathbf{1 9 8 6}$ & $\mathbf{1 9 8 9}$ & $\mathbf{1 9 9 4}$ & $\mathbf{1 9 9 9}$ \\
\hline 6551 & 6223 & 6232 & 6227 & 623071 & 623071 & 612020 & 612020 & 612007 \\
\hline 6552 & 6234 & 6233 & 6228 & 623074 & 623074 & 623071 & 623071 & 612020 \\
\hline 6554 & 6236 & 6235 & 6229 & 623075 & 623075 & 623074 & 623074 & 612022 \\
\hline & & & & & & 623075 & 623075 & 623071 \\
\hline & & & & & & & & 623073 \\
\hline & & & & & & & & 623074 \\
\hline & & & & & & & & 623075 \\
\hline & & & & & & & & 623079 \\
\hline
\end{tabular}

\begin{tabular}{|c|c|c|c|c|c|c|c|c|}
\hline \multicolumn{10}{|c|}{ Actividades seleccionadas para la construcción del sector de la } \\
información en México \\
\hline $\mathbf{1 9}$ \\
\hline $\mathbf{1 9 6 1}$ & $\mathbf{1 9 6 6}$ & $\mathbf{1 9 7 1}$ & $\mathbf{1 9 7 6}$ & $\mathbf{1 9 8 1}$ & $\mathbf{1 9 8 6}$ & $\mathbf{1 9 8 9}$ & $\mathbf{1 9 9 4}$ & $\mathbf{1 9 9 9}$ \\
\hline 3614 & 2811 & 2811 & 2911 & 342001 & 3420 & & 342001 & 341033 \\
\hline 3966 & 2812 & 2812 & 2912 & 342002 & 3823 & & 342002 & 341034 \\
\hline & 3722 & 3641 & 3640 & 342003 & 3832 & & 382302 & 342001 \\
\hline & 3723 & 3721 & 3721 & 342004 & & & 383201 & 342002 \\
\hline
\end{tabular}


78 InvestigacoónBiblictedoógia v. 18 N o. 37 julio/ diciembre de 2004

\begin{tabular}{|c|c|c|c|c|c|c|c|c|}
\hline \multicolumn{9}{|c|}{$\begin{array}{l}\text { Actividades seleccionadas para la construcción del sector de la } \\
\text { información en México }\end{array}$} \\
\hline \multicolumn{9}{|c|}{$\begin{array}{l}\text { Censos industriales } \\
\text { (Cont.) }\end{array}$} \\
\hline 1961 & 1966 & 1971 & 1976 & 1981 & 1986 & 1989 & 1994 & 1999 \\
\hline & & & & 383201 & & & 383206 & 352234 \\
\hline & & & & 383203 & & & 385007 & 383201 \\
\hline & & & & 383204 & & & & 383202 \\
\hline & & & & 383204 & & & & 383204 \\
\hline & & & & 383205 & & & & 383205 \\
\hline & & & & 383206 & & & & 343206 \\
\hline & & & & 385006 & & & & 385006 \\
\hline & & & & 385007 & & & & 385007 \\
\hline
\end{tabular}

\begin{tabular}{|c|c|c|c|c|c|c|c|c|}
\hline \multicolumn{7}{|c|}{ Actividades seleccionadas para la construcción del sector de la } \\
información en México \\
\hline $\mathbf{1 9 6 1}$ & $\mathbf{1 9 6 6}$ & $\mathbf{1 9 7 1}$ & $\mathbf{1 9 7 6}$ & $\mathbf{1 9 8 1}$ & $\mathbf{1 9 8 6}$ & $\mathbf{1 9 8 9}$ & $\mathbf{1 9 9 4}$ & $\mathbf{1 9 9 9}$ \\
\hline 8122 & 8122 & 8311 & 8201 & 832001 & 840001 & 831113 & 831113 & 811056 \\
\hline 8123 & 8123 & 8312 & 8412 & 832002 & 840002 & 831121 & 831121 & 811061 \\
\hline 8312 & 8311 & 8313 & 8413 & 832003 & 840003 & 831122 & 831122 & 811062 \\
\hline 8313 & 8312 & 8314 & 8421 & 822004 & 840004 & 921102 & 921101 & 812011 \\
\hline 8314 & 8313 & 8315 & 8422 & 832008 & 840008 & 921103 & 921102 & 812021 \\
\hline 8315 & 8314 & 8316 & 8423 & 832013 & 840013 & 921104 & 921103 & 812031 \\
\hline 8316 & 8315 & 8622 & 8425 & 832015 & 840015 & 921105 & 921104 & 831113 \\
\hline 8318 & 8316 & 8812 & 8512 & 833113 & 840016 & 921106 & 921105 & 831121 \\
\hline 8319 & 8319 & 8813 & 8821 & 833132 & 850013 & 921107 & 921106 & 831122 \\
\hline 8713 & 8812 & 8917 & 8824 & 833133 & 850032 & 921108 & 921107 & 921101 \\
\hline 8714 & 8813 & 8922 & 8825 & 931101 & 850033 & 921108 & 921108 & 921102 \\
\hline 8724 & 8922 & 8951 & 8922 & 931102 & 931101 & 921110 & 921110 & 921103 \\
\hline & 8947 & & 8941 & 931103 & 931102 & 921111 & 921111 & 921104 \\
\hline & & & 9112 & 931104 & 931102 & 922100 & 922100 & 921105 \\
\hline & & & 9113 & 931105 & 931103 & 941101 & 941101 & 921106 \\
\hline & & & 9114 & 931106 & 931104 & 941104 & 941104 & 921107 \\
\hline & & & 9115 & 931107 & 931105 & 941105 & 941105 & 921108 \\
\hline & & & 9119 & 931108 & 931106 & 941107 & 941107 & 921109 \\
\hline
\end{tabular}


Situación, proceso y construcción de la sociedad de la información ...

\begin{tabular}{|c|c|c|c|c|c|c|c|c|}
\hline \multicolumn{9}{|c|}{$\begin{array}{l}\text { Actividades seleccionadas para la construcción del sector de la } \\
\text { información en México } \\
\text { (Cont.) }\end{array}$} \\
\hline \multicolumn{9}{|c|}{ Censos de servicios } \\
\hline 1961 & 1966 & 1971 & 1976 & 1981 & 1986 & 1989 & 1994 & 1999 \\
\hline & & & 9121 & 931109 & 931107 & 941203 & 941201 & 921110 \\
\hline & & & 9122 & 932100 & 931110 & 942101 & 941203 & 921111 \\
\hline & & & 9191 & 941101 & 931111 & 951002 & 942101 & 922100 \\
\hline & & & 9199 & 941104 & 941101 & 951003 & 951002 & 925003 \\
\hline & & & & 941105 & 941104 & 951004 & 951003 & 941101 \\
\hline & & & & 941107 & 941105 & 951008 & 951004 & 941102 \\
\hline & & & & 942101 & 941107 & 951014 & 951008 & 941103 \\
\hline & & & & 951202 & 942101 & 951017 & 951014 & 941104 \\
\hline & & & & 959002 & 959007 & & 951016 & 941105 \\
\hline & & & & 959003 & & & 951017 & 941106 \\
\hline & & & & & & & & 941107 \\
\hline & & & & & & & & 942101 \\
\hline & & & & & & & & 951001 \\
\hline & & & & & & & & 951002 \\
\hline & & & & & & & & 951003 \\
\hline & & & & & & & & 951004 \\
\hline & & & & & & & & 951005 \\
\hline & & & & & & & & 951006 \\
\hline & & & & & & & & 951007 \\
\hline & & & & & & & & 951009 \\
\hline & & & & & & & & 951010 \\
\hline & & & & & & & & 951013 \\
\hline & & & & & & & & 951014 \\
\hline & & & & & & & & 951016 \\
\hline & & & & & & & & 951017 \\
\hline & & & & & & & & 952002 \\
\hline & & & & & & & & 952007 \\
\hline & & & & & & & & 961105 \\
\hline
\end{tabular}


80 Investigacón Biblidtedoógica v. 18 N o. 37 julio/ diciembre de 2004

\begin{tabular}{|c|c|c|c|c|c|c|c|c|}
\hline \multicolumn{7}{|c|}{ Actividades seleccionadas para la construcción del sector de la } \\
información en México
\end{tabular}

Elrubrocorrespondientealainformaciónagrupalasactividades relacionadascon lainformaciónrealiza das enlaunidadproductiva:investiga ción, dirección, software, análisis de mercados y otras, cuya finalidad es organizar la sociedad (educación, salud, investigación, consultorías, comunicaciones, transacciones comerciales, burocraciapúblicayprivada, organizacióndereglamentosycontroldelasociedad, etcétera). Todo ello representa la complejidad de la estructura social en la cual se han emplea do lastecnolo gías delain formación y co mu nicación en sus respec tivoscampos, ademásdelempleodepersonalpro fesionalycapacitado.

Des de el en fo que de Clark, México se halla en la terce ra eta pa; o en el sec torservicios sirecurrimos ala pers pec tiva de Bell, en virtud de que su par ti cipa ción en elPIB y el porcentaje de personal contratado sobrepasa a los sectores agrícola e industrial has ta fi na les de los años no ven ta. De acuer do con los da tos de los cen sos eco nó micos, constatamos que, efectivamente, nuestro país se encuentra en un proceso de construcción deunaso ciedaddelain formación. Peroparade sarrollarlacreemosque elgo bierno debe brin darasis ten cia y los fondos ne ce sarios, pues dichares pon sa bilidad también debería recaer enins titu ciones mul tina cio nales deca pital priva do para evitarquegrandessectores delapoblaciónqueden fueradeesteproyecto. 


\section{CONCLUSIONES}

Enlasúl ti mas déca das hemossido tes tigos deuna aceleradasuce sióndeavances tecnológicos aplica dos alain forma ciónyla comunicación, quehan propicia doque estesectorse es tu die des de diversaspers pectivas.La socie dad delain formaciónse carac te riza porhabervalo ra do alain formación comosu principal fuerza producti va, lo cual su po nela adop ción de un nuevo ciclo in dus trial y de una nue va di ná mica económica.

Paracon formarunasocie dad de in forma ción, algu nos paísescomo Es tados Unidosde América, Fran cia,Ja pón yla Co munidadEu ropea, en treotros, han es tablecido po líticas y pla nes que han sido apo ya dos tan to por el go bierno como por la ini ciativa privada, y también han establecido prioridades para aplicar las tecnologías de in formación. Estosmismos ejemplos deberían aplicarseen México, paraimpulsarla construccióndeunasociedaddeinformación.

Elincrementodel PIBy de fuen tes de em pleo han mejo ra dola eco no mía de países que has ta aho ralle van la de lan te ra de tal modo que al gu nos au to res, como Marc Porat,Da nielBelly Ma nuel Cas tells en tre otros, men cio nanla con for ma ción de un nuevo sectoreconómico con formadoporactividadesrelaciona das conlainformación. Para elcaso denuestropaís, estas actividades dein formación comienzanaregistrarse en los censos a partir de los años sesenta, pero fue hasta la década de los noventa cuandoés tas seincrementanatalgra do que esposible representarlas comouncuar to sectoreconómico.

Laconstrucción desociedades dein formaciónenpaíses desarrolla doshapermitidocambiosenlaestructuraeconómica,política,educativaetcétera, enbeneficio de la so cie dad, por lo que nues tro país no debeque daral margen de esos cam bios, pues de una u otra manera mediante el fenómeno de globalización formamos parte de un mismosistema.

Aunque en Méxicosehancreadomecanismos deíndolegubernamentalcomolos Planes de de sarrolloin formático1995-2000y2001-2006, ademásdelos de corteaca démico,comolasinvestiga ciones realiza das porelCUIB-UNAM en fo cadas a la so ciedaddelconocimien to, fal tan trabajos quele den con tinuidadaesta te má ticaenáreas comolain dustriadelain forma ciónyla merca do tecnia en México quenosin diquena cienciaciertaen queáreas tene mos mayo res opor tu nida des yde bilidadesparadis mi nuir nuestra dependencia de países desarrollados y reducir la brecha existente en comparaciónconéstos.

\section{FUENTES}

Bell,Daniel. ElAdverimientodelaSociedadPost-industrial:UnIntentodeProgn sis Sacial. Madrid: Alianza, 1976.

- -. La tecnoloǵadelacomunicacón: parabienoparamal.Harvard:Biblioteca

Harvard de Administración de Empresas, 1979. 
Bello Orta,JoséMa nuel. “La so cie dad delain forma ción”. México:Facul tad de Filosofía y Letras, UNAM, 1996.

Braman, Sandra. "Information Policy and the United States Supreme Court". University of Minessota, 1988, tesis de doctorado.

- -. "Defining Information: An Approach for Policymakers", en Tdeo munications Pdigy(septiembre de 1989): 233-242.

Carras cosa,JoséLuis. Informadón delaeaindustrialalasodiedaddelainforma iớn Madrid: Espasa-Calpe, 1991.

Cas tells,Ma nuel. HighTedhndogy,SpacandSociły.Los Án ge les: Sage, 1985.

- -. Ladiudadinformacional:teonloǵasdlainformacón restnucturacóneconómi caye procesourbanorejonal. Madrid: Alianza, 1995.

- - TheRiseof theNework Sodity. Londres: Blackwell, 1996.

- -. "Entender nuestro mundo", en Reista deOcidenteno. 205 (mayo de 1998): 113-145.

Castells, Manuel đtal Nuevas temdoǵas, ๔comáa ysocedaden España Madrid: Alianza, 1986.

Castells Manuel y Yuko Aoya ma. "Paths Towards the In forma tio nal Society: Employment Structure in G-7 Contries, 1920-1990”, en Intemational Labar Reviewvol. 133, no. 1 (1994): 5-33.

Cawkell, A.E. Evdution of anInformationSocidy. Londres: ASLIB, Association for Information Management Information House, 1987.

Cazadero, Manuel. Lasreduainesindustriales México: Fondo de Cultura Económica, 1996.

CEPAL. AméicaLatina comerioexterior segúnla dasificaciónindustrialintemaconal unifomedetodas las adividades económicas(CIIU). Santiago de Chile: CEPAL, 1993.

Clark, Co lin.TheConditionsofEconomicProgess Lon dres:Macmillan, 1940.

Dirección GeneraldeEsta dística. IV Censocomeraial1961. Resumengeneral. México: SIC, 1961.

- -. V Censo comerial 1966. Resumengeneal. México: SIC, 1961.

- - VI Censo comerial. Resumen geneal. 1971. México: SIC, 1971.

- -. VIICensocomerial. Resumengeneral 1976. México:Secreta ría deProgramación y Presupuesto, 1976.

- -. VIII Censo comerial. Resumen general. 1981. Aguascalientes: INEGI, 1981.

- -. VII Censoindustrial. Resumengeneral 1961. México: SIC, 1961.

- - VIII Censoindustrial. Resumengeneral 1966: México: SIC, 1966.

- -. IX Censoindustrial. Resumengeneral 1971: México: SIC, 1971.

- -. X Censoindustrial. Resumengeneral 1976. México: Se cre ta ría de Programación y Presupuesto, 1979. 
Dirección General de Estadística. VIII Censodetranspatesycommicadiones 1976 México: Secretaría de Programación y Presupuesto. 1976.

- - IV Censodeservicios Resumengeneral 1961. México: SIC, 1961.

- -. V Censodeserviaios Resumengeneral 1961. México: SIC, 1966.

- -. VI Censodeservicios Resumengeneral 1971. México: SIC, 1971.

- -. VII Censodeserviaios Resumengenal 1975. México: Secretaría de Programación y Presupuesto, 1976.

Drucker, Pe ter Fer di nand.Post-capitalistSodidy. Nue va York: Har per Bussines, 1993.

Drucker, P.E. "The Next Revolution of the Information", en Forbes ASAP, 24 de agosto de 1998, pp. 47-58.

Fo res ter, Tom. Sociedaddealtatecnoloǵa. lahistoniadelarevoluaióndelateanole ǵa delainfomadón México: Siglo XXI, 1992.

Giddens, Anthony. Conseunenias dela modemidad Madrid: Alianza, 1994.

Gó mez Ro ble do, Ma ría Eu ge nia. "El pa pel de la in for ma ción en una sociedad en desarrollo”, en Revista Interamericana deBiblideedoǵa vol. 7, nos. 1-2, pp. 55-66.

INEGI. Méxiøoyy. Aguascalientes: INEGI, 1991.

- -. EstadísticashistáricasdaMéxi@g tomo 2, Aguas ca lien tes:INEGI, 1999.

- - Sistema decuentas nadionales deMéxica Cuentas debienes yserviaos, 1988 1996 tomo I. Aguascalientes: INEGI, 1999.

--. Clasificaciónmexicanadkadividadesyproductos1999. Aguas calientes: INEGI, 1999.

- -. X Censo comerial resultados definitivos, 1989. Aguascalientes: INEGI, 1989.

--. XICensocomercial. Censoseconómicos1994.Aguascalientes: INEGI, 1989.

--. XICensoindustrial. Resumengeneral1981. Aguascalientes: INEGI, 1981.

- -. XIII Censo industrial. Resumen general 1989. Aguascalientes: INEGI, 1989.

- -. XIV Censo industrial. Industrias manufactureras, extractivas y detricidad Aguascalientes: INEGI, 1994.

- -. IX Censo detransportes y commicaciones 1981. Aguascalientes: INEGI, 1981.

- -. X Censo de transportes y commicaiones 1985. Aguascalientes: INEGI, 1986.

- -. XI Censo detranspates y comnicaaiones 1989. Aguascalientes: INEGI, 1989.

- -. XII Censodetranspates y comunicadions 1994 Aguascalientes: INEGI, 1994.

- -. VII Censodeserviaios, 1981. Aguascalientes: INEGI, 1980. 
INEGI. VIII Censodeserviaios, 1986. Aguascalientes: INEGI, 1986.

- - IX Censodeservicios, 1986. Aguascalientes: INEGI, 1986.

- -. X Censodeserviaos, 1989. Aguascalientes: INEGI, 1989.

- -. XI Censodeserviáos,1994. Aguascalientes: INEGI, 1984.

Jeon, Dong Y. "On the Scene: The Nature of the Information Sector in the Information Society: An Economic and Societal Perspective", en Spetial Libranies (verano de 1990): 25-29.

Kuhlmann,Federico. Commicadionespasadyfuturos México:Secre ta ría de Comunicaciones y Transportes, 1989.

Lafuente López, Ramiro. Endumbralde cambia lastecnoloǵasdelainforma dón yla commicadón México: Centro Universitario de Investigaciones Bibliotecológicas, UNAM, 1997.

Machlup, Fritz. TheProductionandDistributionofKnoweelgeintheUnitedSta tes Nueva Jersey: Princenton University Press, 1971.

Martin, Sta na B. "In forma tion Te chnology, Em ployment, and the In formation Sector: Trends in Information Employment 1970-1995", en Journal otheAmericanSodityforInformationSaiencevol. 49, no. 12, 1998, p. 1053-1069.

Masuda, Yomeji. La sociedad infomatizada como sociedad postindustrial, Madrid: Fundesco-Tecnos, 1984.

Morales Campos, Es tela.Lainformaciónendiniciodelaeradectrónica, vol. 2. México: CUIB, UNAM, 1998.

PoderEjecutivoFederal. PlanNacionaldeDesamdlo1995-2000. Progamade DesamdloInfomáticoMéxico:Secreta ría de Ha cien dayCrédito Publico, 1995.

Po rat, Marc Uri. "De fi ning an In for ma tion Sec tor in the U.S. Economy", en Information RepartsandBibliographiesvol. 5, no. 5 (1976): 17-31.

- - ThelnformationEconmy:DefinitionandMeesurement Wa shing ton, D.C.: U.S.: Department of Commerce, 1977.

Schement,Jorge Reina. "Porat, Belland the In formation So ciety Reconside red: The Growth of In for ma tion Work in the Ear ly Twen tieth Century", en Infomation Processing \& Managment, vol. 26, no. 4 (1990): 449-465.

Stam back, Tho mas M.Æal. ServicioslanuevaeconomíaMéxico:Poligrafics, 1984.

UNCTAD.Méxicounaeconomáadeservicios RepartedlproyeetoMex/ 87/ 026/ . Nueva York: UNCTAD-Secretaría deComercioy Fo men toIn dus trial, 1991.

Za vala Alardín, Gon za lo.Lasodiedadinformatizada: ¿unanuevautopía?. Mé xico: Trillas, 1990.
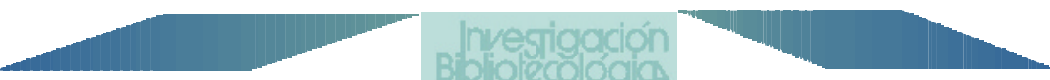